\title{
The influence of the explosion Mechanism on the Fe-group ejecta of core collapse supernovae
}

\author{
Carla Fröhlich $^{1}$, F.-K. Thielemann ${ }^{1}$, G. Martínez Pinedo ${ }^{2}$, \\ and M. Liebendörfer ${ }^{3}$ \\ ${ }^{1}$ Department of Physics and Astronomy, University of Basel, CH-4056 Basel, Switzerland \\ email: carla.froehlich@unibas.ch \\ ${ }^{2}$ ICREA and Institut d'Estudis Espacials de Catalunya Universitat Autònoma de Barcelona, \\ E-08193 Barcelona, Spain \\ ${ }^{3}$ Canadian Institute for Theoretical Astrophysics, University of Toronto, \\ Toronto ON M5S 3H8, Canada
}

\begin{abstract}
Core collapse supernovae are responsible for at least half of the galactic inventory of Fe-group elements and probably for most of the Fe-group abundances seen in metal poor stars. Recent simulations show the emergence of a proton-rich $\left(Y_{e}>0.5\right)$ region in the innermost ejected mass zones due to the neutrino interaction with matter. We explore the nucleosynthesis implications of these findings that result in enhanced abundances of ${ }^{45} \mathrm{Sc},{ }^{49} \mathrm{Ti}$, and ${ }^{64} \mathrm{Zn}$, which is consistent with chemical evolution studies and observations of low metallicity stars.
\end{abstract}

Keywords. Nucleosynthesis, neutrinos, stars: supernovae: general

\section{Introduction}

Understanding the mechanism of core collapse supernovae has been an open issue for many years. It is generally accepted that a massive star proceeds through all burning stages (from H to Si burning), ending with the collapse of the Fe-core to nuclear densities and the formation of a neutron star. However, the details of the explosion mechanism are still not fully understood. In the last decade, different routes were followed to explore the explosions driven by energy deposition through neutrino and antineutrino capture reactions on free neutrons and protons $\left(\nu_{e}+n \rightarrow p+e^{-}\right.$and $\left.\bar{\nu}_{e}+p \rightarrow n+e^{+}\right): 1$. Convective instabilities but with still simplified neutrino transport. 2. Improved neutrino transport schemes (full solution of the Boltzmann transport equation). All of these approaches lead (in one way or the other) to higher neutrino luminosities (e.g. Keil, Janka \& Mueller 1996) or higher energy deposition efficiency in the convective regions (e.g. Mayle \& Wilson 1988, Herant et al. 1994, Mezzacappa et al. 2001). However, with currently known physics spherically symmetric (1D) radiation-hydro calculations do not explode, not even by the delayed neutrino heating mechanism (Rampp \& Janka 2000, Mezzacappa et al. 2001, Liebendörfer et al. 2001, Janka et al. 2003, Thompson et al. 2003). In rotational symmetry (2D) the situation is not much better: radial neutrino transport approximations favor explosions which are not confirmed by calculations with improved neutrino physics (Mezzacappa et al. 1998, Fryer \& Warren 2002, Buras et al. 2003). Recently, Janka et al. (2005) report on a weak explosion of an $11.2 M_{\odot}$ progenitor using a full $180^{\circ}$ grid (the same simulation using a $90^{\circ}$ grid did not explode).

Despite all the problems with understanding the explosion mechanism, supernova nucleosynthesis has been explored for a long time (Woosley \& Weaver 1995, Thielemann 
et al. 1996, Rauscher et al. 2002, Chieffi \& Limongi 2002, Umeda \& Nomoto 2005). All of these calculations have in common that they are based on artificially induced explosions, employing either a thermal bomb or a piston to explode the progenitor star. In these approaches, the mass cut between the remnant and the ejecta has to be determined by additional conditions (and does not evolve naturally from the simulation). As long as we know the correct amount of energy needed to obtain explosions of the order of $10^{51} \mathrm{erg}$ (as seen in observations) this approach is reasonable for the outer layers. For the innermost layers, however, these methods do not address the effects directly related to the physical processes causing the explosion. In particular, the electron fraction $Y_{e}$ remains unchanged at the value of the progenitor star. This is of concern as the $Y_{e}$ is an important quantity to correctly characterize nucleoynthesis in the innermost layers (undergoing Si burning) and therefore relevant to the Fe-group (Thielemann et al. 1996, Nakamura et al. 1999).

In section 2 we will show how successful explosions with a consistent treatment of $Y_{e}$ can be simulated realistically in the absence of fully self-consistent simulations. In section 3 we will address the effects of a $Y_{e}>0.5$ on the nucleosynthesis in these innermost layers.

\section{Hydrodynamical Simulations}

The investigations presented here were performed in spherical symmetry with general relativistic Boltzmann neutrino transport (Mezzacappa \& Messer 1999, Liebendörfer et al. 2004). The adaptive grid enables us to continue the simulations until the density in the region between the remnant and the ejecta drops to about $\sim 10^{6} \mathrm{~g} / \mathrm{cm}^{3}$. At this point, the simulations are continued with an explicit hydrodynamical code (Bravo et al. 1993) until the temperature falls below $0.2 \times 10^{9} \mathrm{~K}$. In this code, the region inside of the mass cut is replaced by a radiation bubble with a static gravitational potential and is assumed to expand adiabatically. For a more detailed description see Fröhlich et al. (2004).

\subsection{How to obtain an explosion}

Many improvements possibly leading to successful supernova explosions have been discussed and investigated. The net effects are either convective instabilities at the neutrino sphere, or changes in the neutrino luminosity via improved opacities or via convective transport inside the proto-neutron star.

We simulate these effects in two different ways: 1. Enhancing the neutrino luminosity by reducing the neutrino scattering cross sections (while keeping all other reactions at the standard values) or 2. Enhancing the energy deposition efficiencies by increasing the neutrino and antineutrino capture reactions on free neutrons and protons (and their inverse reactions).

These are both not fully self-consistent approaches. Nevertheless, they are an important improvement compared to the traditional piston or thermal bomb approach: First, no external energy has to be added to the system to produce an explosion, and second, the mass cut between the remnant the ejecta emerges consistently from the simulation.

\subsection{Effect of weak interactions}

In all of our simulations the weak interactions play an important role for the explosion. The key reactions are

$$
\begin{aligned}
& \nu_{e}+n \rightleftarrows p+e^{-} \\
& \bar{\nu}_{e}+p \rightleftarrows n+e^{+} .
\end{aligned}
$$


The dominating neutrino and antineutrino capture reactions lead to a resulting $Y_{e}>$ 0.5 (Fröhlich et al. 2004, Pruet et al. 2005). This occurs (at high temperatures) when neutrinos and antineutrinos have very similar spectra favoring antineutrino captures over neutrino captures due to the proton-neutron mass difference. Another characteristic of these ejecta is the relatively high entropy $(\sim 30-50)$. In Figure 1 the electron fraction $Y_{e}$ is shown for different models. The models "A" are models with reduced neutrino scattering cross sections. In the models " $\mathrm{B}$ " the explosions are obtained by increasing the neutrino and antineutrino captures reactions (plus their inverse reactions) in the heating region. From the neutrino spectra (see Figure 2) the two main contributions to the neutrino luminosity can be distinguished: The steeper declining accretion luminosity (dominating at $\sim 250-450 \mathrm{~ms}$ after bounce) and the diffusion luminosity with its slower decline (dominating throughout the rest of the calculation).

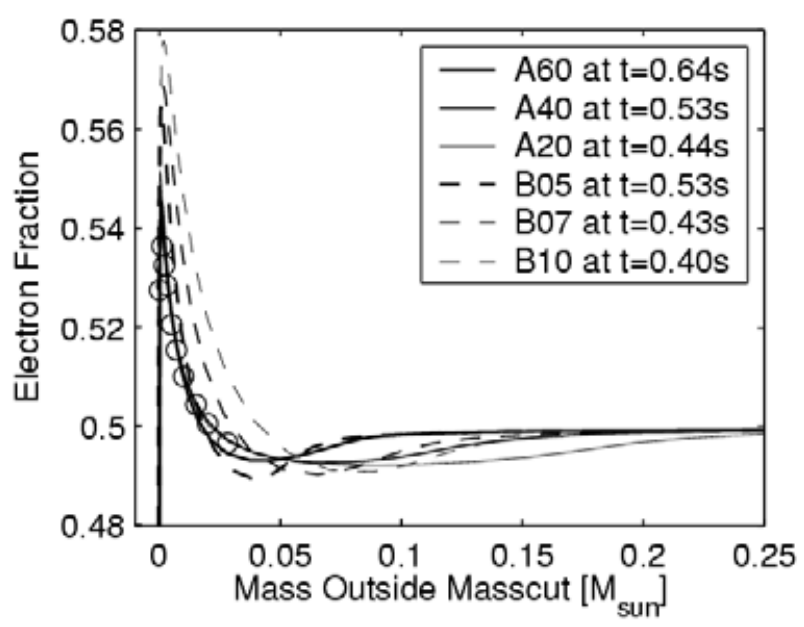

Figure 1. Electron fraction as function of mass at the time when the simulation switches to the explicit hydrodynamical code. (A) Reduction of neutral current scattering opacities. (B) Enhancement of the reaction time scales.

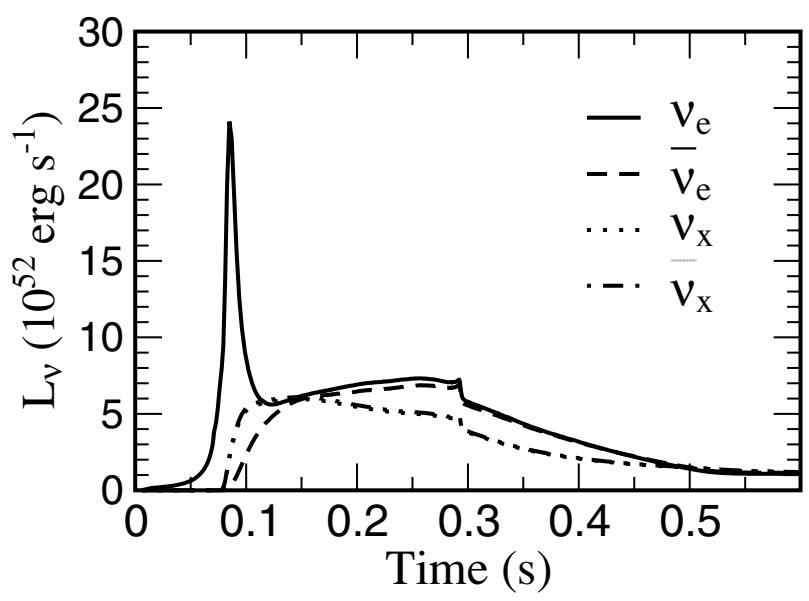

Figure 2. Neutrino and antineutrino luminosities as function of time after bounce. The kink at $\sim 320 \mathrm{~ms}$ after bounce stems from the Doppler shift when the shock passes through $R=500 \mathrm{~km}$. 


\section{Nucleosynthesis}

In this section we will discuss the effect of such "neutrino-driven" explosions (as described in section 2) on the nucleosynthesis predictions. We will show how a resulting $Y_{e}>0.5$ can help to remedy some of the problems with Fe-group abundances. In addition, the question if and how the core collapse supernova environment can contribute to the production of nuclei with $A>64$ is addressed.

We present nucleosynthesis results for exploratory studies of one model (A40, i.e. neutrino scattering cross sections reduced by $40 \%$ ). Only in the innermost few hundredths of a solar mass considered is $Y_{e}>0.5$ attained. Important features of our models are: The mass cut emerges from the simulation (not a free parameter anymore) and the electron fraction $Y_{e}$ is consistently determined from all weak interactions contributing to abundance changes.

The main products in these innermost zones are $\mathrm{H},{ }^{4} \mathrm{He}$, and ${ }^{56} \mathrm{Ni}$, resulting from a proton- and alpha-rich freeze out from quasi-statistical equilibrim (QSE). This remaining hydrogen in the innermost layers is not mixed in but originates directly from these zones. Neutrino-driven explosions ameliorate the problems in the Fe-group predictions as they are found in thermal bomb or piston driven calculations. The overproduction of $\mathrm{Ni}$ isotopes is reduced. Elemental abundances for $\mathrm{Sc}, \mathrm{Cu}$, and $\mathrm{Zn}$ show much better agreement with obersvations of metal-poor and extremely metal-poor stars. On the other hand, vanadium still cannot be explained.

Another interesting feature of the neutrino-driven supernova nucleosynthesis predictions is the production of nuclei with $A>64$. In Figure 3, three different calculations are shown: a thermal bomb explosion (Thielemann et al. 1996), a neutrino-driven explosion without explicitely including neutrino induced reactions in the postprocessing network, and a neutrino-driven explosion where all the neutrino induced reactions contributing to changes in abundances are treated explicitely. In the calculation based on a thermal bomb explosion there is no flux past $A=64$. In neutrino-driven explosions, there is always some flux going beyond $A=64$. The explicit treatment of the reactions $\nu_{e}+n \rightarrow p+e^{-}$and $\bar{\nu}_{e}+p \rightarrow n+e^{+}$makes the difference, allowing the production of nuclei beyond $A=64$ and maybe even of some light p-nuclei. We observe an appreciable production of heavy nuclei, exhibiting an $r p$-process pattern at the waiting-point nuclei $\left({ }^{64} \mathrm{Ge},{ }^{68} \mathrm{Se}{ }^{72} \mathrm{Kr}{ }^{76} \mathrm{Sr}\right.$ decaying to $\left.{ }^{64} \mathrm{Zn},{ }^{68} \mathrm{Zn},{ }^{72} \mathrm{Ge},{ }^{76} \mathrm{Se}\right)$.

Besides the explosion mechanism (neutrino-driven versus piston or thermal bomb) other factors such as the details of the expansion affect the nucleosynthesis beyond $A=64$. We explore the influence of the expansion on the nucleosynthesis by varying the expansion time scale and/or expansion behavior. The expansion is such that matter first expands exponentially and then turns into an outflow with constant velocity. This can be parametrized with three parameters: the time scale for expansion $(\tau)$, the asymptotic speed of matter (related to $\Delta$ ), and a parameter controlling the transition from exponential to linear $\left(\rho_{2}\right)$. In Figure 4 the influence on the nucleosynthesis from varying different parameters is shown for an exploratory mass zone. Note that the abundances shown in Figure 4 are not integrated abundances but rather abundances only for one mass zone. In some cases it is possible to produce nuclei up to strontium or even beyond (light $p$-nuclei). The nucleosynthesis for nuclei with $A>64$ is discussed in details in Fröhlich et al. (2005). 


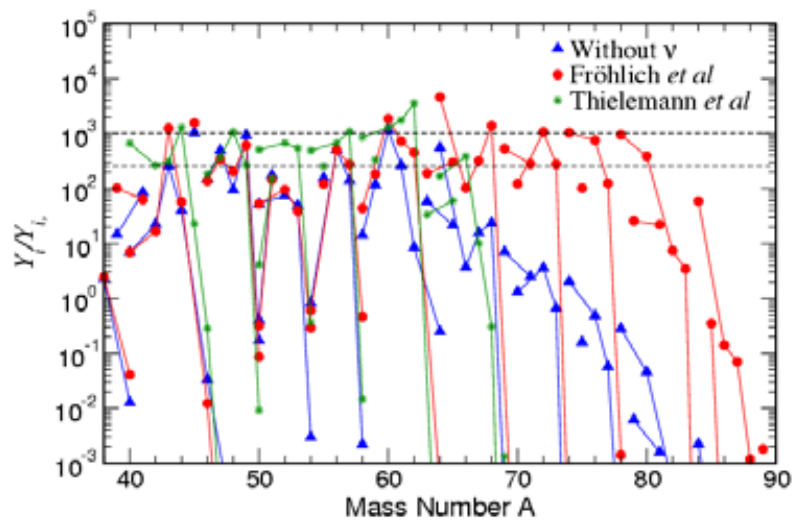

Figure 3. Isotopical abundances as function of mass number. The stars (green) are from TNH96 (thermal bomb calculation). The triangles (blue) and the circles (red) are from neutrino-driven explosions. Explicit treatment of neutrino-induced reactions (red circles) produces an appreciable amount of nuclei with $A>64$.

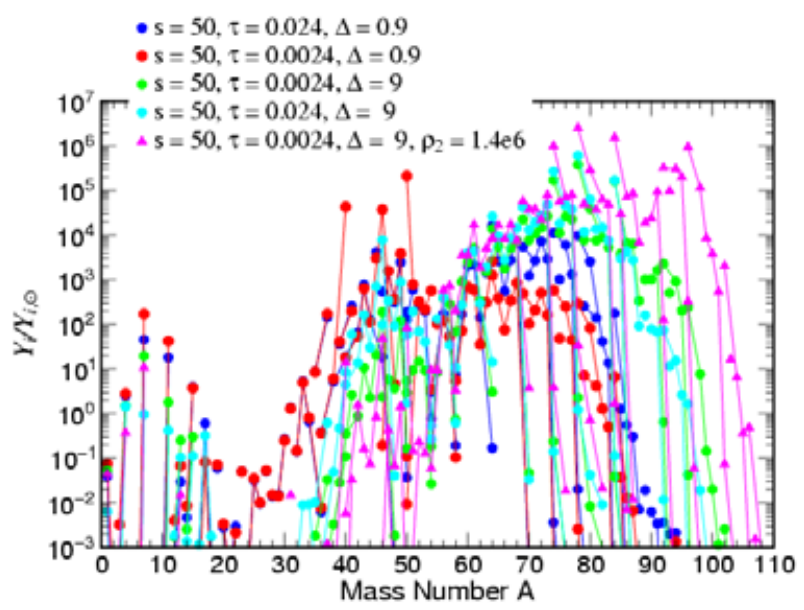

Figure 4. Abundances normalized to solar from varying different parameters in the expansion. Note that the abundances are not integrated abundances over the whole Fe-ejecta but rather abundances for one (typical) mass zone. The quantity $s$ denotes the entropy, $\tau$ is the expansion time scale, and $\Delta$ is related to the asymptotic speed of matter $(\Delta=0.9$ corresponds to a speed of $\sim 1000 \mathrm{~km} / \mathrm{s})$.

\section{Conclusions}

We perform core collapse nucleosynthesis calculations based on explosions and interactions of neutrons and protons with a neutrino flux during the expansion. The explosions are ensured either via reducing the neutrino scattering cross sections or via increasing the neutrinos and antineutrino capture reactions on nucleons in the heating region. Both methods lead to successful explosion with the electron fraction $Y_{e}$ being consistently determined by all weak interactions and a mass cut between remnant and ejecta that emerges from the simulation. Our simulations show $Y_{e}>0.5$, i.e. a proton-rich environment in the innermost layers. The dominant reactions contributing to $Y_{e}>0.5$ are neutrino and antineutrino capture reactions on free nucleons. The main nucleosynthesis products in these layers undergoing complete Si-burning and a proton- and alpha-rich 
freeze-out are $\mathrm{H},{ }^{4} \mathrm{He}$, and ${ }^{56} \mathrm{Ni}$. The nucleosynthesis results for these neutrino-driven explosions (as described above) are in good agreement with observations from metal-poor stars. We find clear improvements in the prediction of the Fe-group elements scandium, copper, and zinc. In addition,we find appreciable production of heavy nuclei with $A>64$ in these neutrino-driven explosions with explicit treatment of neutrino and antineutrino capture reactions in the nuclear reaction network. The details of the nucleosynthesis beyond $A=64$ depend on different factors (for example the expansion details) and is explored in details in Fröhlich et al. (2005).

\section{Acknowledgements}

This work is supported by the Swiss SNF grant 200020-105328.

\section{References}

Bravo, E., Dominguez, I., Isern, J., Canal, R., Hoeflich, P., \& Labay, J. 1993, Astron. Astrophys. J. 269, 187

Buras, R., Rampp, M., Janka, H.-T., \& Kifonidis, K., Phys. Rev. Lett. 90, 241101

Chieffi, A. \& Limongi, M. 2002, Astrophys. J. 577, 281

Fröhlich, C., Hauser, P., Liebendörfer, M., Martínez-Pinedo, G., Thielemann, F.-K., Bravo, E., Zinner, N.T., Hix, W.R., Langanke, K., Mezzacappa, A. \& Nomoto, K. 2004, Astrophys. J. subimtted (astro-ph/0410208))

Fröhlich, C., Martínez-Pinedo, G., Hix, W. R., Langanke, K., Liebendörfer, M., Thielemann, F.-K. \& Zinner, N.T. 2005, in preparation

Fryer, C.L. \& Warren, M.S. 2002, Astrophys. J. Letters 574, L65

Herant, M., Benz, W., Hix, W.R., Fryer, C.L., \& Colgate, S.A. 1994, Astrophys. J. 435, 339

Janka, H.-T., Buras, R., \& Rampp, M. 2003, Nucl. Phys. A 718, 269

Janka, H.-T., Buras, R., Kitaura Joyanes, F.S., Marek, A., Rampp, M., Scheck, L. 2005, Nucl. Phys. A 758, 19

Keil, W., Janka, H.-T., \& Müller, E. 1996, Astrophys. J. Letters 473, L111+

Liebendörfer, M., Mezzacappa, A., Thielemann, F.-K., Messer, O.E.B., Hix, W.R., \& Bruenn, S.W. 2001, Phys. Rev. D 63, 103004

Liebendörfer, M., Messer, O.E.B., Mezzacappa, A., Bruenn, S.W., Cardall, C.Y., \& Thielemann, F.-K. 2004, Astrophys. J. Suppl. 150, 263

Mayle, R. \& Wilson, J.R. 1988, Astrophys. J. 334, 909

Mezzacappa, A., Liebendörfer, M., Messer, O.E.B., Hix, W.R., Thielemann, F.-K., \& Bruenn, S.W. 2001, Phys. Rev. Lett. 86, 1935

Mezzacappa, A., Calder, A.C., Bruenn, S.W., Blondin, J.M., Guidry, M.W., Strayre, M.R., \& Umar, A.S. 1998, Astrophys. J. 495, 911

Mezzacappa, A. \& Messer, O.E.B. 1999, J. Comp. Appl. Maths. 109, 281

Mezzacappa, A. \& Bruenn, S.W. 1993a, Astrophys. J. 405, 637

Nakamura, T., Umeda, H., Nomoto, K., Thielemann, F., \& Burrows, A. 1999, Astrophys. J. 517, 193

Pruet, J., Woosley, S.E., Buras, R., Janka, H.-T., \& Hoffman, R.D. 2005, Astrophys. J. 623, 325

Rampp, M. \& Janka, H.-T. 2000, Astroph. J. Letters 539, L33

Rauscher, T., Heger, A., Hoffman, R.D., \& Woosley, S.E. 2002, Astrophys. J. 576, 323

Thielemann, F.-K., Nomoto, K., \& Hashimoto, M. 1996, Astrophys. J. 460, 408

Thompson, T.A., Burrows, A., \& Pinto, P.A. 2003, Astrophys. J. 592, 434

Umeda, H. \& Nomoto, K. 2005 Astrophys. J. in press

Woosley, S.E. \& Weaver, T.A. 1995, Astrophys. J. Suppl. 101, 181 